\title{
Máscaras caseiras na pandemia de COVID-19: recomendações, características físicas, desinfecção e eficácia de uso*
}

\section{Homemade masks in the COVID-19 pandemic: recommendations, physical characteristics, disinfection and efficacy}

\author{
Mascarillas caseras en la pandemia de COVID-19: recomendaciones, características físicas, \\ desinfección y eficacia de uso
}

\author{
lago Torres Cortês de Sousa' - (1) orcid.org/0000-0002-1931-7644 \\ Aylla Mesquita Pestana' - (1) orcid.org/0000-0002-7494-9900 \\ Larissa Pavanello' - (1) orcid.org/0000-0003-3469-4226 \\ Michelle Franz-Montan' ${ }^{1}$ - (i) orcid.org/0000-0003-0760-1389 \\ Karina Cogo-Müller ${ }^{2}$ - (1) orcid.org/0000-0002-9048-8702 \\ 'Universidade Estadual de Campinas, Faculdade de Odontologia de Piracicaba, Piracicaba, SP, Brasil \\ 2Universidade Estadual de Campinas, Faculdade de Ciências Farmacêuticas, Campinas, SP, Brasil
}

\section{Resumo}

Objetivo: Descrever as recomendações, características físicas, métodos de desinfecção e eficácia de uso de máscaras caseiras na redução da transmissão da COVID-19. Métodos: Realizou-se busca nas bases de dados MEDLINE, SciEL0 e Google Scholar, além das recomendações oficiais de uso. Resultados: Foram incluídas 31 referências. A capacidade de filtração de tecidos variou entre 5\% e 98\%. Tecidos 100\% algodão em duas ou três camadas apresentaram eficácia de filtração entre 70\% e 99\% em estudos in vitro. Máscaras caseiras, cirúrgicas e respiradores apresentaram respirabilidade entre 2,2 e 3,0 Pascal. A capacidade de redução da propagação de microrganismos por pessoas usando máscaras caseiras foi três vezes menor do que usando máscaras cirúrgicas, embora tenha sido superior ao não uso de máscaras. Conclusão: A respirabilidade de máscaras caseiras mostrou-se adequada, enquanto a capacidade de filtração parece ser inferior à das máscaras cirúrgicas, mas superior a não se usar máscara. Não há evidências que respaldem a eficácia e efetividade das máscaras caseiras.

Palavras-chave: Betacoronavírus; Infecções por Coronavírus; Infecções Respiratórias; Máscaras Faciais; Dispositivos de Proteção Respiratória; Revisão.

\footnotetext{
* lago Torres Cortês de Sousa (Processo n 88887.480037/2020-00) e Aylla Mesquita Pestana (Processo n 88887.480042/2020-00) recebem bolsa da Coordenação de Aperfeiçoamento de Pessoal de Nível Superior (Capes)/Ministério da Educação (MEC). Larissa Pavanello é bolsista do Conselho Nacional de Desenvolvimento Científico e Tecnológico (CNPq)/Ministério da Ciência, Tecnologia, Inovações e Comunicações (MCTIC) (Processo n 132616/2020-3).
}

\section{Correspondência:}

lago Torres Cortês de Sousa - Faculdade de Odontologia de Piracicaba, Av. Limeira, n 901, Bairro Areião, Piracicaba, SP, Brasil. CEP: 13414-903.

E-mail: iagocortes20@gmail.com 


\section{Introdução}

A COVID-19 é uma doença infecciosa que afeta principalmente os pulmões, provocada pelo coronavírus 2, causador da síndrome respiratória aguda grave (SARS-CoV-2). Os primeiros registros de infecção ocorreram na China, em dezembro de 2019, e a Organização Mundial da Saúde (OMS) declarou a COVID-19 uma pandemia em 11 de março de $2020 .{ }^{1}$

\section{Medidas não farmacológicas são recomendadas para reduzir a propagação de COVID-19, entre elas o uso de máscaras por toda a população. As máscaras de fabricação caseira, mesmo não classificadas como equipamentos de proteção individual, são também recomendadas.}

Em janeiro de 2021, contavam-se mais de $90 \mathrm{mi}-$ lhões de infectados, mais de 1,5 milhão de mortos e 219 países afetados, Estados Unidos, Índia e Brasil à frente, com o maior número de casos. ${ }^{2,3}$

Medidas não farmacológicas foram recomendadas para reduzir a propagação da doença, entre elas o uso de máscaras por toda a população, incluindo indivíduos assintomáticos. ${ }^{4-8} \mathrm{~A}$ utilização de máscaras de uso profissional, como máscaras cirúrgicas e respiradores, pela população geral, pode resultar na escassez desses materiais para os profissionais na linha de frente do tratamento das pessoas que adoecem. Sendo assim, tem-se recomendado o uso de máscaras de fabricação caseira, mesmo que não sejam classificadas como equipamentos de proteção individual e não tenham passado por testes de controle de qualidade após sua confecção. ${ }^{9,10}$

A presente revisão teve por objetivo descrever as recomendações de uso, características físicas, métodos de desinfecção e eficácia das máscaras caseiras na redução da transmissão da COVID-19.

\section{Métodos}

Trata-se de revisão narrativa da literatura. Foram realizadas pesquisas nas bases de dados MEDLINE (via PubMed), SciEL0 e Google Scholar (100 primeiros resultados), na busca de estudos experimentais in vitro, ensaios clínicos e estudos observacionais. Os termos utilizados nessas pesquisas foram cloth mask, homemade mask, breathability cloth mask, breathability homemade mask, disinfection cloth mask, disinfection bomemade mask e mask, associados ou não ao termo 'COVID-19'. Foram considerados elegíveis estudos que avaliaram as características físicas dos tecidos e máscaras (eficácia de filtração, respirabilidade e influência do ajuste facial na capacidade de filtração), métodos de desinfecção de máscaras caseiras, e eficácia e efetividade do uso de máscaras caseiras.

Também foram realizadas buscas pelas recomendações sobre as máscaras caseiras no Ministério da Saúde do Brasil, Agência Nacional de Vigilância Sanitária (Anvisa), OMS, Centers for Disease Control and Prevention dos Estados Unidos (CDC/EUA) e European Centre for Disease Prevention and Control (ECDC). As buscas foram realizadas até 29 de outubro de 2020 . Três autores (Sousa ITC, Pestana AM e Pavanello L) foram responsáveis pela leitura dos títulos e resumos, para seleção inicial, e leitura dos textos completos, para avaliação da elegibilidade dos estudos identificados. A plataforma Rayyan (https://rayyan.qcri.org) foi utilizada nessas etapas.

\section{Resultados}

Foram recuperados 195 artigos, dos quais 15 foram duplicados e 165, excluídos após leitura do título e resumo, resultando em um total de 15 artigos incluídos. Também foram incluídas 16 referências de instituições oficiais.

\section{Recomendações oficiais}

Agências reguladoras e organizações de saúde recomendaram o uso de máscaras caseiras por toda a população quando houvesse necessidade de sair do isolamento social, sob o argumento de que as máscaras podem funcionar como uma barreira física, diminuindo a dispersão dos vírus por gotículas de saliva e aerossóis a partir do controle da fonte de infecção: indivíduos infectados, assintomáticos ou pré-sintomáticos. ${ }^{4,5,7,-14}$ Segundo a OMS, máscaras de tecido devem conter três camadas: uma externa, de tecido resistente a água (poliéster; híbrido de poliéster e algodão; 
polipropileno); uma interna, com capacidade absorvente de água (como algodão); e uma intermediária, para atuar como filtro (polipropileno ou poliéster). ${ }^{12,13}$ $0 \mathrm{CDC} / \mathrm{EUA}$ indica que a máscara confeccionada em duas camadas não deve permitir a passagem de qualquer luminosidade quando colocada contra uma fonte de luz. ${ }^{14} 0$ Quadro 1 resume as principais indicações de uso de máscaras caseiras, cirúrgicas e respiradores, enquanto o Quadro 2 mostra os tecidos recomendados para a confecção dessas máscaras.

Apesar das recomendações de uso, a $0 \mathrm{MS}^{4}$ e 0 ECDC ${ }^{10}$ alertaram quanto ao risco de contrair SARS-CoV-2 pelo uso e manuseio inadequados das máscaras, falsa sensação de segurança por parte do usuário, além do possível relaxamento de medidas protetoras, como 0 distanciamento social e a higiene das mãos. ${ }^{4,10}$

\section{Capacidade de filtração e de ajuste facial}

A maioria das iniciativas que verificaram a capacidade de filtração de máscaras são estudos in vitro que utilizaram equipamentos geradores de gotículas $(>5 \mu \mathrm{m})$ ou aerossóis $(\leq 5 \mu \mathrm{m})$ através das máscaras ou tecidos, para avaliar seu potencial de bloqueio. ${ }^{15-21}$ Várias substâncias foram utilizadas na produção das partículas, como cloreto de sódio, ${ }^{15,17,18}$ nanopartículas fluorescentes ${ }^{16}$ e partículas de poliestireno, ${ }^{20}$ além de microrganismos como 0 vírus da gripe aviária,$^{18} \mathrm{bac}-$ teriófago MS222 e bactérias da espécie Staphylococcus aureus $^{19} \mathrm{e}$ Bacillus atrophaeus. ${ }^{22}$

Algumas combinações de tecido e camadas mostraram capacidade de filtração mais elevada: máscaras feitas de tecidos $100 \%$ algodão de 600 fios por polegada, em uma ou duas camadas (acima de 98\%); ${ }^{17}$ máscaras de tecidos $100 \%$ algodão de 200 fios por polegada, em uma camada (96\%); ${ }^{16}$ máscaras de tecidos híbridos de algodão e seda, em uma camada (acima de $94 \%),{ }^{17}$ máscaras de $70 \%$ algodão e $30 \%$ poliéster, em uma camada (93\%) ${ }^{16}$ máscaras de tecido não tecido (TNT), em dupla camada $(93 \%) ;{ }^{19}$ e máscaras com uma camada de poliéster e quatro camadas de papel de cozinha (95\%). ${ }^{18}$ Outros tecidos e combinações apresentaram resultados inferiores, como seda natural em uma, duas ou quatro camadas (54\% a $88 \%$ ), algodão de 80 fios por polegada de uma ou duas camadas $(9 \% \text { a } 49 \%)^{14}$ e TNT de uma camada (42\%). ${ }^{19}$ Máscaras cirúrgicas apresentaram eficácia de filtração entre $76 \%$ e $98 \% .^{17-19}$
Ensaios com avaliação de partículas de diferentes tamanhos (20 a $1.000 \mathrm{~nm}$ ) e velocidades de dispersão $(5,5 \mathrm{~cm} / \mathrm{s}$ e $16,5 \mathrm{~cm} / \mathrm{s})$ mostraram que tecidos $100 \%$ algodão, 100\% poliéster e híbridos de algodão com outros tecidos tiveram uma taxa de penetração de partículas (o contrário da filtração) variáveis, entre $40 \%$ e $90 \%$, com aumento da penetração de acordo com 0 aumento da velocidade de ar utilizada. ${ }^{15} \mathrm{~A}$ um fluxo de $8 \mathrm{~L} / \mathrm{min}$ e $19 \mathrm{~L} / \mathrm{min}$, máscaras caseiras com válvulas de plástico e látex tiveram eficácia de filtração acima de $60 \%$, enquanto outras duas máscaras caseiras variaram entre $10 \%$ e $90 \%{ }^{21}$ A um fluxo de $32 \mathrm{~L} / \mathrm{min}$, tecidos de algodão de pesos entre 157 e $360 \mathrm{~g} / \mathrm{m}^{-2}$ mostraram eficiência de filtração entre 5\% e 25\%; nas máscaras de seda, essa eficácia foi de 4\%; nas máscaras cirúrgicas, de $33 \%$; e nos respiradores, de $95 \%{ }^{20}$

Estudo in vitro ${ }^{22}$ para avaliar a capacidade de filtração de tecidos em condições de vedação total mostrou que a capacidade de filtração para aerossóis contendo Bacillus artophaeus foi de 69\% para tecidos 100\% algodão (uma camada), 60\% para linho (uma camada) e 96\% para máscaras cirúrgicas. Já para bacteriófago MS2, a capacidade de filtração foi de 50\% para tecidos 100\% algodão (uma camada), 54\% para linho (uma camada) e $89 \%$ para máscaras cirúrgicas. Tecido $100 \%$ algodão com duas camadas foi testado somente para Bacillus artophaeus e apresentou filtração de $70 \%{ }^{22}$

0 mesmo estudo ${ }^{22}$ simulou o uso das máscaras em pessoas saudáveis $(n=21)$, para verificar a capacidade de bloqueio de dispersão de microrganismos enquanto tossiam duas vezes em uma câmara de amostragem móvel, utilizando máscaras cirúrgicas, caseiras $(100 \%$ algodão) ou nenhuma máscara. A capacidade de redução da propagação de microrganismos pelos voluntários usando máscaras caseiras foi três vezes menor do que quando usaram máscaras cirúrgicas, porém foi superior ao não uso de máscaras. ${ }^{22}$

A influência da vedação também foi avaliada nos ensaios de eficácia de filtração de máscaras caseiras, cirúrgicas e respiradores. ${ }^{17} \mathrm{~A}$ presença de espaços entre a máscara e a face representa uma inadequação de ajuste entre ambos, o que afeta a eficácia da máscara facial. ${ }^{14}$ As máscaras de proteção respiratória de uso não profissional devem cobrir nariz, boca e queixo, a denominada zona de proteção, não devem ter válvulas inspiratórias e/ou expiratórias e devem ser bem ajustadas ao nariz, bochechas e queixo para, adequadas à morfologia do usuário, garantir vedação contra a 
Quadro 1 - Recomendações de agências, órgão e organização de saúde consultados sobre 0 uso de máscaras caseiras, máscaras cirúrgicas e respiradores

\begin{tabular}{|c|c|}
\hline Máscaras caseiras & $\begin{array}{l}\text { População sadia, assintomática ou pré-sintomática em áreas com casos confirmados de COVID-19, quando houver a } \\
\text { necessidade de sair do isolamento. } \text {. }^{47-10,12}\end{array}$ \\
\hline Máscaras cirúrgicas & $\begin{array}{l}\text { Profissionais de saúde na prática clínica, recepcionistas, pessoas com diagnóstico ou sintomas de COVID-19 e } \\
\text { indivíduos com alto risco de enfrentar os estágios mais graves da doença, como idosos e pessoas que apresentam } \\
\text { comorbidades. }{ }^{3-5,7,10,12,38}\end{array}$ \\
\hline Respiradores & $\begin{array}{l}\text { Profissionais que atuam na linha de frente do combate à COVID-19, expostos a procedimentos geradores de aerossol, como } \\
\text { atendimento odontológico, nebulização, manobras de reanimação cardiopulmonar ou procedimentos envolvendo as vias } \\
\text { aéreas. }^{5-7,10,12,38}\end{array}$ \\
\hline \multicolumn{2}{|c|}{$\begin{array}{l}\text { Protetores faciais podem ser usados como opção para pessoas com deficiência mental, transtornos do desenvolvimento e crianças, para as quais o uso de } \\
\text { máscaras não é viável. } \\
\text { Máscaras faciais devem ser evitadas em menores de } 2 \text { anos de idade, pessoas inconscientes e incapazes de remover a máscara sem o auxílio.j,4,9 }\end{array}$} \\
\hline
\end{tabular}

\section{Quadro 2 - Principais tipos de tecidos recomendados para máscaras caseiras}

\begin{tabular}{|c|c|c|}
\hline Instituição ${ }^{\mathrm{a}}$ & Tipo de tecido & $\begin{array}{l}\text { Número de } \\
\text { camadas }\end{array}$ \\
\hline \multirow{4}{*}{ Ministério da Saúde ${ }^{11}$} & De saco de aspirador & \multirow{4}{*}{2} \\
\hline & Híbrido: 55\% poliéster e $45 \%$ algodão & \\
\hline & $100 \%$ algodão & \\
\hline & Fronhas de tecido antimicrobiano & \\
\hline \multirow{3}{*}{ Agência Nacional de Vigilância Sanitária ${ }^{5}$} & $100 \%$ algodão & \multirow{3}{*}{3} \\
\hline & Misturas de algodão com elastano, tendo no mínimo 90\% de algodão & \\
\hline & Tecido não tecido (TNT) & \\
\hline \multirow{6}{*}{ Organização Mundial da Saúde ${ }^{4,12,13}$} & $100 \%$ algodão & \multirow{6}{*}{3} \\
\hline & Poliéster & \\
\hline & Celulose & \\
\hline & Seda & \\
\hline & Nylon & \\
\hline & Polipropileno & \\
\hline Centers of Disease Control and Prevention ${ }^{9}$ & Algodão & 2 \\
\hline
\end{tabular}

a) 0 European Centre for Disease Prevention and Control (ECDC) não apresenta especificação de tecido e número de camadas de máscaras caseiras.

atmosfera ambiente (Figura 1). Elas também devem ser desenhadas de forma a serem colocadas e removidas facilmente, manterem-se fixas no rosto e evitarem aperto excessivo e desconforto durante 0 uso. ${ }^{23}$

Um ensaio com simulação de situações de vedação e não vedação das máscaras e respiradores, para diferentes tamanhos de partículas, encontrou filtração de 99\% (para partículas maiores que 300 $\mathrm{nm})$ a $85 \%$ (para partículas menores que $300 \mathrm{~nm}$ ) para respiradores $\mathrm{N} 95$. A presença de um orifício correspondente a $1 \%$ da área de superfície do respirador reduziu a eficácia de filtração para $34 \%$.
Nas mesmas condições, máscaras cirúrgicas apresentaram filtração de $99 \%$ (partículas >300 nm em situação de vedação), $76 \%$ (partículas $<300 \mathrm{~nm}$ em situação de vedação) e $44 \%$ a $50 \%$ (com a presença de um orifício correspondente a $1 \%$ da área de superfície da máscara, simulando um pequeno desajuste facial). Para máscaras caseiras, como as híbridas de algodão e seda, essa variação foi de 98\% (partículas $>300 \mathrm{~nm}$ ) a 94\% (partículas <300 nm) e 37\% (com a presença de um orifício correspondente a $1 \%$ da área de superfície da máscara, simulando um pequeno desajuste facial). ${ }^{17}$ 


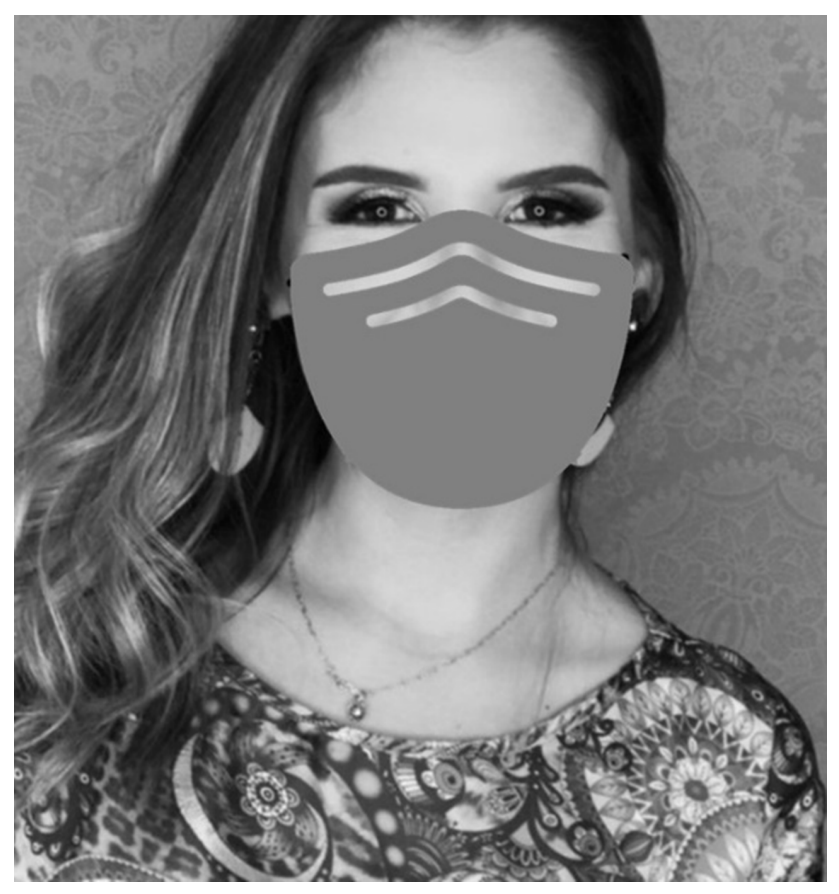

Nota: Máscaras caseiras devem cobrir nariz, boca e queixo (denominada zona de proteção), ajustando-se devidamente à morfologia facial; a presença de válvulas inspiratórias ou expiratórias não é recomendada.

Figura 1 - Zona de proteção da máscara facial de uso não profissional

Máscaras caseiras foram menos eficazes quanto à filtração e ao ajuste facial, quando comparadas às máscaras cirúrgicas e respiradores. ${ }^{24,25}$ Um ensaio avaliou o fator de proteção (FP) calculado, que considera a porcentagem de partículas filtradas pelas máscaras em crianças $(\mathrm{n}=11)$ e adultos $(\mathrm{n}=28) .{ }^{24}$ Esse fator foi obtido considerando-se, inicialmente, a razão da porcentagem da filtração: \%F = (número de partículas no ar dentro da máscara por $\mathrm{cm}^{3} /$ número de partículas no ar fora da máscara por $\mathrm{cm}^{3}$ ) x 100 . Uma vez obtido o valor de \%F, calculou-se o FP, que é o inverso da porcentagem de filtração, dividido por 100 $(\mathrm{FP}=\% \mathrm{~F} / 100)^{-1} .^{24}$

Em adultos, o FP das máscaras (mediana e intervalo interquartil), quando testadas em período curto de uso, foi de 2,5 $(2,1-2,9)$ para máscaras caseiras, 4,1 (3,1 - 7,2) para máscaras cirúrgicas e 113,0 (26,0 - 210,0) para respiradores $\mathrm{N} 95$. Em crianças, o fator de proteção foi de 2,2 $(1,5-2,2)$ para máscaras caseiras, 3,2 (2,2 - 4,1) para máscaras cirúrgicas e 18,0 $(6,1-165,0)$ para respiradores $\mathrm{N} 95 .^{24}$ Em geral, as máscaras caseiras proporcionaram proteção 50 vezes menor do que os respiradores FFP-2 (equivalentes aos respiradores N95) e 25 vezes menor do que as máscaras cirúrgicas, apresentando menor FP nas crianças. ${ }^{24}$

Estudo in vitro avaliou a capacidade de filtração e ajuste de máscaras cirúrgicas $(n=3)$, respiradores N95 (n=3) e máscaras de algodão com duas camadas $(n=10)$ mediante testes de ajuste em um voluntário, utilizando equipamento gerador de aerossol em conjunto com dois contadores de partículas, para quantificar a presença de aerossóis de cloreto de sódio a $2 \%(0,02 \mu \mathrm{m}$ a $1 \mu \mathrm{m})$ fora e dentro da máscara, durante um minuto. Os valores de filtração obtidos foram comparados entre as máscaras e respiradores: máscaras de algodão em duas camadas apresentaram eficácia de filtração inferior a $60 \%$, sendo consideradas menos ajustáveis à anatomia facial, conforme evidenciado pelo teste de ajuste. Já as máscaras cirúrgicas com presença de clipe nasal apresentaram filtração acima de 75\%; e os respiradores N95, considerados equipamentos de proteção individual e certificados para testes de ajuste facial, apresentaram filtração superior a 99\%. ${ }^{25} \mathrm{~A}$ adição de uma fina camada de meia de nylon cobrindo as máscaras caseiras, com o intuito de melhorar 0 ajuste facial e reduzir os espaços entre 0 
rosto e a máscara, resultou em aumento da capacidade de filtração para valores acima de $70 \% .^{25}$

\section{Respirabilidade das máscaras caseiras}

A respirabilidade aceitável de uma máscara cirúrgica deve estar abaixo de 49 Pascal por centímetro quadrado $\left(\mathrm{Pa} / \mathrm{cm}^{2}\right)$. Para as máscaras caseiras, uma diferença de pressão aceitável deve estar abaixo de $100 \mathrm{~Pa} / \mathrm{cm}^{2}{ }^{26}$

A combinação ilimitada de tecidos e materiais resultou em filtração e respirabilidade variáveis, sendo recomendado o uso de pelo menos duas a três camadas de tecidos para máscaras caseiras. $4,5,9,11$

Estudo realizado com 24 crianças entre 8 e 11 anos, ao comparar três tipos de máscaras caseiras destinadas a protegê-las da exposição à poluição do ar, observou que, para todas as máscaras, a respirabilidade foi um dos critérios negativos. As perceber que as máscaras afetavam sua capacidade de respirar, as crianças as removiam durante as atividades de caminhada e corrida. ${ }^{27}$

Um estudo in vitro ${ }^{17}$ avaliou a respirabilidade por meio de pressão diferencial em 15 tipos de tecidos para confecção de máscaras caseiras, e comparou-a com os valores correspondentes encontrados para respiradores e máscaras cirúrgicas. Máscaras caseiras apresentaram valores de filtração e respirabilidade semelhantes aos das máscaras cirúrgicas e respiradores, a depender da constituição dos tecidos: tecidos $100 \%$ algodão, com uma ou duas camadas, apresentaram eficácia de filtração acima de $98 \%$ e respirabilidade de 2,5 Pascal (Pa), resultados bastante semelhantes aos dos respiradores (99\% de filtração e 2,2 Pa de respirabilidade) e máscaras cirúrgicas (99\% de filtração e 2,5 $\mathrm{Pa}$ de respirabilidade). Também apresentaram valores semelhantes os tecidos híbridos de algodão e seda (filtração de $98 \%$ e respirabilidade 3 Pa), algodão, poliéster e elastano (98\% de filtração e 3 Pa de respirabilidade) e algodão e poliéster ( $96 \%$ de filtração e $3 \mathrm{~Pa}$ de respirabilidade). ${ }^{17}$

\section{Desinfecção das máscaras caseiras}

As máscaras devem ser manuseadas com cautela, para evitar contaminação cruzada com outros itens, ${ }^{4}$ lavadas e desinfetadas após cada uso. ${ }^{28}$ Quanto à higienização das máscaras caseiras, são sugeridas lavagens com água quente ${ }^{4,7,23}$ ou água fria, ${ }^{11,23,28-30}$ utilizando-se detergente ou sabão (Quadro 3); ${ }^{4,5,7,11,28}$ detergente enzimático também foi indicado no processo de lavagem, como alternativa ao detergente comum. ${ }^{23}$ Algumas organizações recomendaram a imersão da máscara em solução com hipoclorito de sódio, por 5 a 30 minutos, ${ }^{5,11,28}$ ou cloro por $1 \mathrm{~min}^{4}{ }^{4} 0$ uso da máquina de lavar com água quente $\left(60^{\circ} \mathrm{C}\right)$ é sugerido, além de uso de ferro de passar após secagem ${ }^{5}$ (Quadro 3).

Um estudo in vitro realizado em 2020 testou em triplicata métodos físicos utilizando calor úmido (vaporizador de água, como uma panela de cozinhar arroz, por 15 minutos) e calor seco (em forno, $100^{\circ} \mathrm{C}$ por 15 minutos), para avaliar a desinfecção de máscaras caseiras, respiradores $\mathrm{N} 95$ e máscaras cirúrgicas, após terem suas superfícies externas ou internas inoculadas com Staphylococcus aureus resistente à meticilina (MRSA) e bacteriófago MS2 ${ }^{31} 0$ método de calor úmido reduziu mais de 5 logaritmos de MRSA e bacteriófago MS2, enquanto o calor seco não resultou em queda superior a 3 logaritmos, independentemente do tipo de máscara. Não foram verificados danos nas máscaras após cinco ciclos de descontaminação. ${ }^{31}$

\section{Eficácia e efetividade de uso na prevenção de infecções respiratórias}

Em 2011, um ensaio clínico randomizado, com 1.607 médicos e enfermeiros de 15 hospitais do Vietnã, observou que a incidência de infecção respiratória foi estatisticamente maior no grupo de máscaras caseiras, comparado ao de máscaras cirúrgicas. ${ }^{32} \mathrm{~A}$ infecção foi confirmada pela deteç̧ão de sintomas clínicos ou por teste laboratorial, por meio da reação em cadeira da polimerase em tempo real mediada por transcrição reversa (RT-qPCR), para 17 vírus respiratórios, incluindo vírus da síndrome respiratória aguda grave - não o SARS-CoV-2.

Estudo observacional realizado em Hong Kong, no período de 31 de dezembro de 2019 (dia 1, sem casos confirmados em Hong Kong) a 9 de abril de 2020 (com 961 casos confirmados), comparou o uso médio de máscaras faciais e o número de casos confirmados laboratorialmente, entre Hong Kong e alguns países representativos na América do Norte, Europa e Ásia. 0 diagnóstico de COVID-19 foi feito por RT-qPCR e a monitoração de uso de máscaras por contabilização do número de usuários em três dias consecutivos (10.500 pessoas foram monitoradas). ${ }^{33} 0$ uso médio de máscaras faciais em Hong Kong foi de 96\%, e 
Quadro 3 - Recomendações sobre lavagem e desinfeç̧ão de máscaras caseiras

\begin{tabular}{|c|c|}
\hline Instituição & Recomendações de lavagem e desinfecção \\
\hline Organização Mundial da Saúde ${ }^{4}$ & $\begin{array}{l}\text { Água a } 60^{\circ} \mathrm{C}+\text { sabão/detergente ou água a temperatura ambiente com sabão/ } \\
\text { detergente e posterior fervura, ou imergir a máscara em cloro a 0,1\% por } 1 \text { minuto e } \\
\text { enxaguar em água à temperatura ambiente. }\end{array}$ \\
\hline Centers for Disease Control and Prevention ${ }^{28}$ & $\begin{array}{l}\text { Imergir a máscara em solução com hipoclorito de sódio a 2,0-2,5\% e água potável } \\
\text { à temperatura ambiente, por } 5 \text { minutos, com enxágue em água à temperatura } \\
\text { ambiente, e secar com ar quente ou ao ar livre; na lavagem em máquinas de lavar, } \\
\text { utilizar sabão e água quente. }\end{array}$ \\
\hline European Centre for Disease Preventions and Control ${ }^{7}$ & Detergente comum a $60^{\circ} \mathrm{C}$. \\
\hline Ministério da Saúde ${ }^{11}$ & $\begin{array}{l}\text { Imergir a máscara em solução com hipoclorito de sódio a 2,0-2,5\% e água potável na } \\
\text { proporção de 1:50, por } 30 \text { minutos. Enxaguar em água corrente e lavar com água e } \\
\text { sabão. Finalizar, após a secagem, com ferro quente. }\end{array}$ \\
\hline Agência Nacional de Vigilância Sanitária ${ }^{5}$ & $\begin{array}{l}\text { Lavagem prévia com água corrente e sabão neutro. Imergir a máscara em solução } \\
\text { de hipoclorito de sódio a } 2,5 \% \text { (ou outro desinfetante equivalente) e água potável } \\
\text { por } 20 \text { a } 30 \text { minutos. Enxaguar em água corrente e finalizar, após a secagem, com } \\
\text { ferro quente. Em máquina de lavar, programar o ciclo completo de lavagem com } \\
\text { temperatura de lavagem a } 60^{\circ} \mathrm{C} \text {. }\end{array}$ \\
\hline
\end{tabular}

a taxa de transmissão da COVID-19, de 129 pessoas infectadas por 1 milhão de habitantes, ao passo que, na Coreia do Sul, a adesão às máscaras faciais foi de $50 \%$, e a proporção de infectados, de 200,5 por 1 milhão de habitantes. ${ }^{33}$ Outros países, como Espanha (2.983,2 infectados/1 milhão de hab.) e Itália (2.250,8 infectados/1 milhão de hab.), tiveram taxas de infectados/1 milhão de hab. mais elevadas. No entanto, os dados de porcentagem de uso de máscaras por ambos os países europeus não foram disponibilizados, somente a informação de que se tratava de populações de regiões onde o uso de máscaras faciais não foi universalmente adotado. ${ }^{33}$

\section{Discussão}

A utilização de máscaras caseiras na prevenção da COVID-19 tem sido recomendada pelas agências, órgão e organização de saúde consultados, em situações nas quais existe necessidade de sair do isolamento social. Tecidos de máscaras caseiras podem apresentar filtração adequada, embora variável, e sempre inferior às máscaras cirúrgicas. Estudos de avaliação do uso de máscaras caseiras durante a pandemia causada pelo SARS-CoV-2 ainda são escassos e pouco conclusivos. Observou-se possível redução na incidência de infecção com o uso de máscaras caseiras. No que diz respeito aos métodos de desinfecção, a lavagem com água e sabão, bem como o uso de solução de água com hipoclorito a 2,0-2,5\%, são as principais ações recomendadas para higienização das máscaras.

As recomendações de uso de máscaras caseiras apresentadas, levam em consideração apenas as publicações oficiais de cinco instituições de saúde (Anvisa, Ministério da Saúde do Brasil, CDC/EUA, ECDC e 0MS), o que é uma limitação para esta revisão. Ademais, não foram encontrados estudos que avaliassem a capacidade de filtração ou eficácia de métodos de desinfecção de máscaras caseiras utilizando-se o SARS-CoV-2: as conclusões sobre a eficácia de filtração e desinfecção das diferentes máscaras pautaram-se nos resultados de estudos que simularam - inanimadamente - 0 SARS-CoV-2, ou que utilizaram outros vírus e bactérias.

As agências, órgão e organização de saúde recomendaram, principalmente, tecido $100 \%$ algodão, ${ }^{4,9,5,11}$ TNT $^{5}$ e tecido híbrido de algodão e poliéster ${ }^{11}$ para confecção de máscaras caseiras. Esses tecidos mostraram-se eficazes in vitro quanto à capacidade de filtração de partículas em aerossóis, apresentando taxas de filtração entre $93 \%$ e $98 \%{ }^{16,17,19}$ Entretanto, pesquisas metodologicamente semelhantes ${ }^{15,20,21}$ apresentaram resultados diferentes, com valores de filtração abaixo de 40\%. Porém, não está clara, nesses estudos, ${ }^{15,20,21}$ a quantidade de camadas ou mesmo a constituição dos tecidos que as compõem. De forma geral, tecidos em dupla camada mostraram-se superiores na filtração, comparados a tecidos com uma única camada. ${ }^{17-19}$ Além do tipo de tecidos das máscaras, 
variações nos experimentos de eficácia de filtração, a exemplo do tamanho das partículas em aerossóis, velocidade de fluxo de ar empregado e vedação ou não da máscara, podem influenciar os resultados, dificultando a comparação entre estudos. ${ }^{16-22}$

A respirabilidade dos tecidos, assim como a capacidade de ajuste facial das máscaras caseiras, são fatores que influenciam a eficácia de filtração das máscaras. ${ }^{27}$ Máscaras caseiras têm potencial de apresentar respirabilidade adequada, semelhantemente às máscaras cirúrgicas e respiradores, ${ }^{16,17,22}$ embora sua capacidade de ajuste facial possa ser inferior, ${ }^{21,22}$ sendo importante ressaltar que máscaras caseiras, da mesmo forma que as cirúrgicas, não são desenhadas para promover vedação, como acontece com os respiradores. A presença de espaço entre o rosto e a máscara, ainda que ajustada, pode reduzir a eficácia de filtração. 17,21,22

Água, sabão e/ou detergentes e desinfetantes como o hipoclorito, são os produtos mais recomendados para desinfecção das máscaras caseiras. ${ }^{4,7,9,11,23}$ Métodos químicos e físicos de desinfecção, como uso de etanol a $70 \%,{ }^{34}$ hipoclorito de sódio ${ }^{35}$ e inativação por calor, ${ }^{36}$ também demonstraram, in vitro, potencial para redução de SARS-CoV-2, apesar de não terem sido usados em máscaras caseiras. ${ }^{37}$ Não se encontrou estudos que avaliassem o impacto dos métodos de desinfecção nos parâmetros físicos das máscaras caseiras, embora se tenha sugerido um número de reutilização e lavagem das máscaras de até 30 vezes. ${ }^{5}$ Estudos que avaliem a capacidade de desinfecção das máscaras, principalmente pela contaminação pelo SARS-CoV-2, ainda são necessários.

Quanto à efetividade de uso das máscaras caseiras na redução da transmissão do SARS-CoV-2 na população, estudos de predição baseados em modelos matemáticos estimaram que o uso de máscaras, mesmo com baixa eficácia de filtração, pode reduzir em cerca de $45 \%$ as mortes causadas pela COVID-19, numa projeção de dois meses. ${ }^{38-40}$ Um estudo observacional, realizado em 2020, concluiu que o uso de máscaras reduziu significativamente a propagação do SARS-CoV-2 na população, ${ }^{33}$ porém, não distinguiu os tipos de máscaras utilizados, tampouco levou em consideração a influência de outros fatores, como cuidados de higiene e distanciamento social. Em ambiente hospitalar, profissionais em uso de máscaras caseiras foram mais susceptíveis a infecções respiratórias, quando comparados a profissionais que usaram máscaras cirúrgicas. ${ }^{32}$ Cumpre observar que fatores como o manuseio e o tempo de uso das máscaras caseiras, ou o risco de infecções oriundas de fora do ambiente hospitalar, não foram controlados.

Em conclusão, a eficácia de filtração e respirabilidade dos tecidos com que são confeccionadas as máscaras caseiras é variável e, em algumas situações, comparável à de máscaras cirúrgicas. No entanto, em situações de uso e sob influência de fatores como a capacidade de ajuste, a máscara caseira pode promover filtração e proteção contra infecções respiratórias inferiores às observadas nas máscaras cirúrgicas e respiradores, embora superiores à não utilização. Métodos de desinfecção com água e sabão e/ou detergentes, ou com desinfetantes, como o hipoclorito, são recomendados mesmo sem evidências científicas de sua eficácia em máscaras caseiras. São necessários mais estudos para avaliar os métodos de desinfecção, eficácia e efetividade de uso das máscaras caseiras na prevenção da COVID-19.

\section{Agradecimentos}

Ao Espaço da Escrita da Pró-Reitoria de Pesquisa/ Unicamp, pelos serviços de tradução de idioma.

\section{Contribuição dos autores}

Sousa ITC e Cogo-Müller K participaram na concepção do estudo. Sousa ITC, Pestana AM, Pavanello L, Franz-Montan M e Cogo-Müller K participaram da análise e interpretação dos dados, redação e revisão crítica relevante do conteúdo intelectual do manuscrito. Todos os autores aprovaram a versão final do manuscrito e são responsáveis por todos os aspectos do trabalho, incluindo a garantia de sua precisão e integridade. 


\section{Referências}

1. Ministério da Saúde (BR). 0 que é COVID-19? [Internet]. Brasília, DF: MS. 2020 [acesso 29 ago. 2020]. Disponível em: https://coronavirus. saude.gov.br/sobre-a-doenca ${ }^{\#} 0$-que-e-covid

2. World Health Organization. WHO coronavirus disease (COVID-19) dashboard [Internet]. [Geneva]: WHO; 2020 [acesso 29 out. 2020]. Disponível em: https://covid19.who.int/

3. World Health Organization. Coronavirus disease (COVID-19) pandemic [Internet]. [Geneva]: WHO; 2020 [acesso 29 ago. 2020]. Disponível em: https://www.who. int/emergencies/diseases/novel-coronavirus-2019

4. World Health Organization. Coronavirus disease (COVID-19) advice for the public [Internet]. [Geneva]: WHO; 2020 [acesso 29 ago. 2020]. Disponível em: https:/www.who.int/emergencies/ diseases/novel-coronavirus-2019/advice-for-public

5. Agência Nacional de Vigilancia Sanitária. Orientações gerais: máscaras faciais de uso não profissional [Internet]. Brasília, DF: Anvisa; 3 abr. 2020 [acesso 29 ago. 2020]. Disponível em: https://www.anvisa.gov.br

6. Centers for Disease Control and Prevention. Coronavirus disease 2019 (COVID-19) [Internet]. [Highway]: CDC; 2020 [acesso 29 ago 2020]. Disponível em: https:/www.cdc.gov/coronavirus/2019ncov/prevent-getting-sick/prevention.html

7. European Centre for Disease Prevention and Control. Considerations for infection prevention and control measures on public transport in the context of COVID-19 [Internet]. [Solna]: ECDC;29 Apr 2020 [acesso 29 ago. 2020]. Disponível em: https://www. ecdc.europa.eu/en/publications-data/covid-19prevention-and-control-measures-public-transport

8. Ministério da Saúde (BR). Como se proteger [Internet]. [Brasília, DF]: MS; 2020 [acesso 29 ago 2020]. Disponível em: https://coronavirus. saude.gov.br/sobre-a-doenca\# ${ }^{\#}$ como-se-proteger

9. Centers for Disease Control and Prevention. How to make masks [Internet]. Centers for disease control and prevention. [Highway]: CDC; 2020 [acesso 29 ago. 2020]. Disponível em: https://www. cdc.gov/coronavirus/2019-ncov/prevent-gettingsick/how-to-make-cloth-face-covering.html

10. European Centre for Disease Prevention and Control. Infographic: using face masks in the community [Internet]. [Solna]: ECDC; 2020 [acesso 29 ago. 2020]. Disponível em: https:// www.ecdc.europa.eu/en/publications-data/ infographic-using-face-masks-community

11. Ministério da Saúde (BR). Nota informativa $n$. 3/2020-CGGAP/DESF/SAPS/MS [Internet]. [Brasília, DF]: MS; 2020 [acesso 29 ago. 2020]. Disponível em: https:/www.saude.gov.br/images/pdf/2020/ April/04/1586014047102-Nota-Informativa.pdf

12. World Health Organization. Advice on the use of masks in the context of COVID-19: Interim guidance [Internet]. [Geneva]: WHO; 6 April 2020 [acesso 29 ago.2020]. Disponível em: https:// apps.who.int/iris/handle/10665/331693

13. World Health Organization. Mask use in the context of COVID-19: Interim guidande [Internet]. [Geneva]: WHO; 1 December 2020 [acesso 14 mar.2020]. Disponível em: https:// apps.who.int/iris/handle/10665/337199

14. Centers for Disease Control and Prevention. Type of masks: cloth masks [Internet]. [Highway]: CDC; 19 Apr 2021 [acesso 14 mar. 2021]. Disponível em: https:/www.cdc.gov/coronavirus/2019-ncov/ prevent-getting-sick/types-of-masks.html

15. Rengasamy S, Eimer B, Shaffer RE. Simple respiratory protection: evaluation of the filtration performance of cloth masks and common fabric materials against $20-1000 \mathrm{~nm}$ size particles. Ann Occup Hyg. 2010;54(7):789-98. doi: https://doi.org/10.1093/annhyg/meq044.

16. Aydin 0, Emon B, Cheng S, Hong L, Chamorro LP, Saif TA. Performance of fabrics for homemade masks against the spread of COVID-19 through droplets: a quantitative mechanistic study. Extreme Mech Lett. 2020;40:100924. doi: https://doi.org/10.1016/j.eml.2020.100924.

17. Konda A, Prakash A, Moss GA, Schmoldt M, Grant GD, Guha S. Aerosol filtration efficiency of common fabrics used in respiratory cloth masks. ACS Nano. 2020 May 26;14(5):6339-47. doi: https://doi.org/10.1021/acsnano.0c03252.

18. Ma XQ, Shan H, Zhang H-L, Li G-M, Yang R-M, Chen J-M. Potential utilities of mask: wearing and instant hand hygiene for fighting SARS-CoV-2. J Med Virol. 2020;92(9):1567-71. doi: https://doi.org/10.1002/jmv.25805.

19. Wang D, You Y, Zhou X, Zong Z, Huang H, Zhang H, et al. Selection of homemade mask materials for preventing transmission of COVID-19: a laboratory 
study. PLoS One. 2020 0ct 15;15(10): e0240285.doi: http://dx.doi.org/10.1371/journal.pone.0240285.

20. Zhao M, Liao L, Xiao W, Yu X, Wang H, Wang Q, et al. Household materials selection for homemade cloth face coverings and their filtration efficiency enhancement with triboelectric charging. Nano Lett. 2020 Jul 8;20(7):5544-52. doi: http:// dx.doi.org/10.1021/acs.nanolett.0c02211.

21. Shakya KM, Noyes A, Kallin R, Peltier RE. Evaluating the ef fi cacy of cloth facemasks in reducing particulate matter exposure. J Expo Sci Environ Epidemiol. 2017;27(3):352-357. doi: http://dx.doi.org/10.1038/jes.2016.42.

22. Davies A, Thompson K-A, Giri K, Kafatos G, Walker J, Bennet A. Testing the Efficacy of homemade masks : would they protect in a influenza pandemic? Disaster Med Public Health Prep. 2020;7(4):413-8. doi: http://dx.doi.org/ 10.1017/dmp.2013.43.

23. Associação Brasileira de Normas Técnicas. Máscaras de proteção respiratória de uso não profissional: guia de requisitos básicos para métodos de ensaio, fabricação e uso [Internet]. Rio de Janeiro: ABNT; 2020 [acesso 29 ago. 2020]. Disponível em: http:// www.cvs.saude.sp.gov.br/up/Guia_0994632_ ABNT_Pratica_Recomendada_para_Mascaras_ de_Uso_Nao_Profissional_compressed (3).pdf

24. van der Sande M, Teunis P, Sabel R. Professional and home-made face masks reduce exposure to respiratory infections among the general population. PLoS One. 2008 Jul 9;3(7):e2618. doi: http:// dx.doi.org 10.1371/journal.pone.0002618. .

25. Mueller A, Fernandez L. Assessment of fabric masks as alternatives to standard surgical masks in terms of particle filtration efficiency. medRxiv 2020.04.17.20069567; doi: https://doi.org/10. 1101/2020.04.17.20069567. Now published in Matter. doi: 10.1016/j.matt.2020.07.006

26. Jung H, Kim JK, Lee S, Lee J, Kim J, Tsai P, et al. Comparison of Filtration efficiency and pressure drop in anti-yellow sand masks, quarantine masks, medical masks, general masks, and handkerchiefs. Aerosol Air Qual Res. 2014;14(3):991-1002. doi: https://doi.org/10.4209/aaqr.2013.06.0201.

27. Smart NR, Horwell CJ, Smart TS, Galea KS. Assessment of the wearability of facemasks against air pollution in primary school-aged children in London. Int $J$ Environ Res Public Health. 2020 Jun 2;17(11):3935. doi: https://doi.org/10.3390/ijerph17113935.
28. Centers for Disease Control and Prevention. How to wash masks [Internet]. [Highway]: CDC; 2020 [acesso 31 ago. 2020]. Disponível em: https://www. cdc.gov/coronavirus/2019-ncov/prevent-gettingsick/how-to-wash-cloth-face-coverings.html

29. Agência Nacional de Vigilancia Sanitária. Nota técnica n. 26/2020/SEI/COSAN/GHCOS/DIRE3/ ANVISA. Recomendações sobre produtos saneantes que possam substituir o álcool 70\% na desinfecção de superfícies, durante a pandemia da COVID-19 [Internet]. [Brasília, DF]: ANVISA; 2020 [acesso 29 ago. 2020]. Disponível em: http://portal. anvisa.gov.br/documents/219201/4340788/ SEI_ANVISA+-+0964813+-+Nota+Técnica. pdf/71c341ad-6eec-4b7f-b1e6-8d86d867e489

30. Agência Nacional de Vigilancia Sanitária. Nota técnica n. 47/2020/SEI/COSAN/GHCOS/DIRE3/ANVISA. Recomendações sobre produtos saneantes que possam substituir o álcool 70\% e[m] desinfecção de objetos e superfícies, durante a pandemia de COVID-19 [Internet]. [Brasília, DF]: ANVISA; 2020 [acesso 29 ago. 2020]. Disponível em: http://portal.anvisa.gov. br/documents/219201/4340788/Nota+Técnica+47. pdf/242a3365-2dbb-4b58-bfa8-64b4c9e5d863

31. Li DF, Cadnum JL, Redmond SN, Jones LD, Donskey CJ. It's not the heat, it's the humidity: effectiveness of a rice cooker-steamer for decontamination of cloth and surgical face masks and $\mathrm{N} 95$ respirators. Am J Infect Control. 2020;48(7):854-5. doi: https://doi.org/10.1016/j.ajic.2020.04.012.

32. MacIntyre CR, Seale H, Dung TC, Hien NT, Nga PT, Chughtai AA, et al. A cluster randomised trial of cloth masks compared with medical masks in healthcare workers. BMJ Open. 2015 Apr 22;5(4):e006577. doi: https:/doi.org/10.1136/bmjopen-2014-006577.

33. Cheng VC-C, Wong S-C, Chuang VW-M, So SY-W, Chen JH-K, Sridhar S, et al. The role of community-wide wearing of face mask for control of coronavirus disease 2019 (COVID-19) epidemic due to SARS-CoV-2. J Infect. 2020;81(1):107-14. doi: https://doi.org/10.1016/j.jinf.2020.04.024.

34. Leslie RA, Zhou SS, Macinga DR. Inactivation of SARS-CoV-2 by commercially available alcoholbased hand sanitizers. Am J Infect Control. 2021;49(3):401-402. doi: https://doi.org/10.1016/j. ajic.2020.08.020. Epub 2020 Aug 18.

35. Chan K-H, Sridhar S, Zhang RR, Chu H, Fung AY-F, Chan G, et al. Factors affecting stability and infectivity of SARS-CoV-2.J Hosp Infect. 
2020;106(2):226-31. doi: https://doi.org/10.1016/j. jhin.2020.07.009. Epub 2020 Jul 9.

36. Auerswald H, Yann S, Dul S, In S, Dussart P, Martin $\mathrm{NJ}$, et al. Assessment of inactivation procedures for SARS-CoV-2. bioRxiv 2020.05.28.120444. doi: https://doi.org/10.1101/2020.05.28.120444

37. Kampf G, Todt D, Pfaender S, Steinmann E. Persistence of coronaviruses on inanimate surfaces and their inactivation with biocidal agents. J Hosp Infect. 2020;104(3):246-51. doi: https://doi.org/10.1016/j.jhin.2020.01.022.

38. Ngonghala CN, Iboi E, Eikenberry S, Scotch M, Maclntyre RC, Bonds MH, et al. Mathematical assessment of the impact of non-pharmaceutical interventions on curtailing the 2019 novel Coronavirus. Math Biosci. 2020;325:108364. doi: https:/doi. org/10.1016/j.mbs.2020.108364. Epub 2020 May 1.

39. Eikenberry SE, Mancuso M, Iboi E, Phan T, Eikenberry $\mathrm{K}$, Kuang Y, et al. To mask or not to mask: modeling the potential for face mask use by the general public to curtail the COVID-19 pandemic. Infect Dis Model. 2020 Apr 21;5:293-308. doi: https://doi. org/10.1016/j.idm.2020.04.001. eCollection 2020.

40. Ministério da Saúde (BR). Saúde e segurança do trabalhador [Internet]. [Brasília, DF]: MS; 2020 [acesso 29 ago. 2020]. Disponível em: https://coronavirus.saude.gov.br/ saude-e-seguranca-do-trabalhador-epi

\section{Abstract}

Objective: To describe the recommendations, physical characteristics, disinfection methods and efficacy of the use of homemade face masks to reduce COVID-19 transmission. Methods: We searched MEDLINE, SciELO and Google Scholar, in addition to the official recommendations for the use of masks. Results: Thirtyone references were included. Fabric filtration efficiency ranged from 5\% to 98\%. The filtration efficiency of three layered $100 \%$ cotton fabric face masks ranged from $70 \%$ and $99 \%$ in vitro studies. Homemade, surgical and respirator masks showed breathability between 2.2 and 3.0 Pascal. The capacity to reduce the spread of microorganisms by people wearing homemade face masks was three times lower when compared to those wearing surgical masks, although this capacity was bigher when compared to those who did not wear masks. Conclusion: The breathability of homemade masks proved to be adequate, while the filtration efficiency seemed to be lower than that of surgical masks, but it was better than not wearing any masks at all. There is no evidence to support the efficacy and effectiveness of homemade masks.

Keywords: Betacoronavirus; Coronavirus infections; Respiratory Infections; Face Masks; Respiratory Protective Equipment; Review.

\section{Resumen}

Objetivo: Describir las recomendaciones, características físicas, métodos de desinfección y efectividad de mascarillas caseras para reducir la transmisión de COVID-19. Métodos: La búsqueda se realizó en las bases de datos MEDLINE, SciELO y Google Scholar, además de las recomendaciones oficiales de uso. Resultados: Se incluyeron 31 referencias. La capacidad de filtración de los tejidos varió entre 5\% y 98\%. Los tejidos al 100\% de algodón, en dos o tres capas, mostraron eficiencia de filtración entre $70 \%$ y $99 \%$, en estudios in vitro. Mascarillas caseras, quirúrgicas y de respiradores mostraron respirabilidad entre 2,2 y 3,0 Pascal. La capacidad de reducir la propagación de microorganismos por personas que usan máscarillas caseras fue tres veces menor que cuando usaban máscarillas quirúrgicas, pero superior a no usarlas. Conclusión: La respirabilidad de las mascarillas caseras puede ser adecuada, mientras que la eficiencia de filtración parece ser inferior a la de las mascarillas quirúrgicas, pero superior a no utilizar mascarilla. No bay evidencia que respalde su eficacia y efectividad.

Palabras clave: Betacoronavirus; Infecciones por Coronavirus; Infecciones del Sistema Respiratorio; Máscarillas Faciales; Dispositivos de Protección Respiratoria; Revisión. 